\title{
On unified-entropy characterization of quantum channels
}

\author{
Alexey E. Rastegin \\ Department of Theoretical Physics, Irkutsk State University, Gagarin Bv. 20, Irkutsk 664003, Russid*
}

\begin{abstract}
We consider properties of quantum channels with use of unified entropies. Extremal unravelings of quantum channel with respect to these entropies are examined. The concept of map entropy is extended in terms of the unified entropies. The map $(q, s)$-entropy is naturally defined as the unified $(q, s)$-entropy of rescaled dynamical matrix of given quantum channel. Inequalities of Fannes type are obtained for introduced entropies in terms of both the trace and Frobenius norms of difference between corresponding dynamical matrices. Additivity properties of introduced map entropies are discussed. The known inequality of Lindblad with the entropy exchange is generalized to many of the unified entropies. For tensor product of a pair of quantum channels, we derive two-side estimating of the output entropy of a maximally entangled input state.
\end{abstract}

PACS numbers: 03.65.-a, 03.67.Hk, 02.10.Ud

Keywords: extremal unravelings, quantum channels, map entropy, additivity properties, $(q, s)$-entropy exchange

\section{INTRODUCTION}

The concept of entropy is one of most important notions in both statistical physics and information theory. New applications of this concept are connected with those advantages that can be reached by using quantum resources to process and transmit the information [1]. In addition to the Shannon and von Neumann entropies, which are both fundamental, other entropic measures were found to be useful. Among them, the Rényi and Tsallis $q$-entropic functionals are well known [2]. A general treatment of these and some other entropies in terms of unified $(q, s)$ entropies was given in Ref. [3]. For many or even all values of parameters $q$ and $s$, the quantum unified $(q, s)$-entropy enjoy features similar to properties of the standard von Neumann entropy [4]. The unified entropies was applied for treatment of quantum entanglement and monogamy [5].

Entropic measures have widely been adopted in studying features of quantum channels [1, 2]. Different characteristics are properly ensured by entropies of different kinds and forms. The entropy exchange [6] and the map entropy [7] have been put to describe entanglement transmission and decoherence induced by a quantum channel. Additivity properties of map entropies with respect to the tensor product of two channels are of interest [8]. For the minimum output entropy, this question is considered to be even more relevant [9, 10]. Together with the von Neumann entropy, the quantum Rényi entropy has been applied for these purposes. In the present work, we treat characteristics of quantum channels with use of the unified $(q, s)$-entropies.

The paper is organized as follows. The main definitions and the notation are introduced in Section II In Section III. we examine those channel unravelings that are extremal with respect to the unified entropies. Continuity estimates of Fannes type are derived for the map $(q, s)$-entropies in Section IV] A distance between rescaled dynamical matrices are quantified by means of both the trace and Frobenius norms. Section $\nabla$ is devoted to properties of the map $(q, s)$ entropies with respect to the tensor product of two quantum channels. Using an extension of Lindblad's inequality, we derive a two-sided estimate on the output $(q, s)$-entropy for the tensor product of two channels acting on maximally entangled input state. Section VI concludes the paper.

\section{DEFINITIONS AND NOTATION}

Let $\mathcal{H}$ be $d$-dimensional Hilbert space. We denote the space of linear operators on $\mathcal{H}$ by $\mathcal{L}(\mathcal{H})$ and the set of positive semidefinite operators on $\mathcal{H}$ by $\mathcal{L}_{+}(\mathcal{H})$. The support of an operator is defined as the vector space orthogonal to its kernel. A density operator $\rho \in \mathcal{L}_{+}(\mathcal{H})$ has unit trace, i.e. $\operatorname{tr}(\rho)=1$. For $\mathrm{X}, \mathrm{Y} \in \mathcal{L}(\mathcal{H})$, we define the Hilbert-Schmidt inner product by [11]

$$
\langle\mathrm{X}, \mathrm{Y}\rangle_{\mathrm{hs}}:=\operatorname{tr}\left(\mathrm{X}^{\dagger} \mathrm{Y}\right)
$$

*Electronic address: rast@api.isu.ru 
The Schatten norms, which form an important class of unitarily invariant norms, are defined in terms of singular values. Recall that singular values $\varsigma_{j}(\mathrm{X})$ of operator $\mathrm{X}$ are put as eigenvalues of $|\mathrm{X}|=\sqrt{\mathrm{X}^{\dagger} \mathrm{X}}$. For $q \geq 1$, the Schatten $q$-norm of $\mathrm{X} \in \mathcal{L}(\mathcal{H})$ is then defined by [11, 12]

$$
\|\mathrm{X}\|_{q}=\left(\sum_{j=1}^{d} \varsigma_{j}(\mathrm{X})^{q}\right)^{1 / q}
$$

This definition is closely related to the $q$-mean $\mathfrak{M}_{q}(x)=\left(\frac{1}{n} \sum_{j=1}^{n} x_{j}^{q}\right)^{1 / q}$. The properties of such means are extensively considered in the book [13]. The family (2.2) includes the trace norm $\|\mathrm{X}\|_{1}$ for $q=1$, the Frobenius norm $\|\mathrm{X}\|_{2}$ for $q=2$, and the spectral norm $\|\mathrm{X}\|_{\infty}=\max \left\{\varsigma_{j}(\mathrm{X}): 1 \leq j \leq d\right\}$ for $q=\infty$. The trace norm distance is one of most frequently used distances. Its partitioned varieties are also be defined on the base of Ky Fan's norms [14, 15]. In some respects, however, we will prefer the Frobenius norm distance. Since the Frobenius norm is induced by the HilbertSchmidt inner product, i.e. $\|\mathrm{X}\|_{2}^{2}=\langle\mathrm{X}, \mathrm{X}\rangle_{\mathrm{hs}}$, this distance is often named the Hilbert-Schmidt distance. Recently, it has found to be fruitful in studying two-dimensional projections of the set of mixed state [16].

The formalism of quantum operations provides a unified treatment of possible state change in quantum theory [1, 11]. Consider a linear map $\Phi$ that takes elements of $\mathcal{L}(\mathcal{H})$ to elements of $\mathcal{L}\left(\mathcal{H}^{\prime}\right)$, and also satisfies the condition of complete positivity. Let id ${ }^{R}$ be the identity map on $\mathcal{L}\left(\mathcal{H}_{R}\right)$, where the space $\mathcal{H}_{R}$ is assigned to ancillary reference system. The complete positivity implies that $\Phi \otimes \mathrm{id}^{R}$ transforms a positive operator into a positive operator again for each dimension of the extended space. Such linear maps are typically called "quantum operations" [1] or "superoperators" [11]. Each completely positive map can be written in the operator-sum representation. Namely, for any $\mathrm{X} \in \mathcal{L}(\mathcal{H})$ we have

$$
\Phi(\mathrm{X})=\sum_{j} \mathrm{~A}_{j} \mathrm{XA}_{j}^{\dagger}
$$

where Kraus operators $\mathrm{A}_{j}$ map the input space $\mathcal{H}$ to the output space $\mathcal{H}^{\prime}$ [1, 11]. In general, the normalization condition implies that

$$
\sum_{j} \mathrm{~A}_{j}^{\dagger} \mathrm{A}_{j} \leq \mathbb{1}
$$

where $\mathbb{1}$ is the identity operator on $\mathcal{H}$. In most of applications, the input and output spaces are the same. When the physical process is deterministic, the equality in Eq. (2.4) holds and $\operatorname{tr}(\Phi(\rho))=1$. In this case, the map $\Phi$ is usually referred to as "quantum channel" [1]. Probabilistic operations, such as probabilistic cloning [17] or quantum state separation [18], are of interest. But in the present paper, we will deal only with deterministic quantum operations.

As an entropic measure, we will use the unified $(q, s)$-entropy introduced in Ref. [3] and further studied in Ref. [4]. The $(q, s)$-entropies form a family of two-parameter entropic functionals continuous with respect to both the parameters [3]. Many generalized entropies including the Rényi and Tsallis ones are contained in this family. The quantum unified $(q, s)$-entropy of density operator $\rho$ is defined as 3$]$

$$
\mathrm{H}_{q}^{(s)}(\rho):=\frac{1}{(1-q) s}\left\{\left[\operatorname{tr}\left(\rho^{q}\right)\right]^{s}-1\right\}
$$

for $q>0, q \neq 1$ and $s \neq 0$. For $q=1$, this entropy is defined as the von Neumann entropy $\mathrm{H}_{1}(\rho)=-\operatorname{tr}(\rho \ln \rho)$. For $s=1$, we obtain the quantum Tsallis $q$-entropy

$$
\mathrm{T}_{q}(\rho):=\frac{1}{1-q} \operatorname{tr}\left(\rho^{q}-\rho\right)=\operatorname{tr}\left(\eta_{q}(\rho)\right),
$$

where $\eta_{q}(x):=\left(x^{q}-x\right) /(1-q)=-x^{q} \ln _{q} x$ in terms of $q$-logarithm $\ln _{q} x=\left(x^{1-q}-1\right) /(1-q)$. In the limit $s \rightarrow 0$, the definition (2.5) leads to the quantum Rényi $q$-entropy $\rho$ defined as

$$
\mathrm{R}_{q}(\rho):=\frac{1}{1-q} \ln \left[\operatorname{tr}\left(\rho^{q}\right)\right] .
$$

For $q=1$, both the expressions (2.6) and (2.7) recover the von Neumann entropy. The classical entropies can all be obtained by replacing the traces with the proper sums over a probability distribution. Let $X$ be random variable, taking $m$ possible values with probabilities $p_{X}(i)(i=1, \ldots, m)$. Its $(q, s)$-entropy is

$$
H_{q}^{(s)}(X):=\frac{1}{(1-q) s}\left[\left(\sum_{i=1}^{m} p_{X}(i)^{q}\right)^{s}-1\right]
$$


for $q>0, q \neq 1$ and $s \neq 0$, and the Shannon entropy $H_{1}(X)=-\sum_{i} p_{X}(i) \ln p_{X}(i)$ for $q=1$. For $s=1$, the right-hand side of Eq. (2.8) gives the classical Tsallis $q$-entropy $T_{q}(X)$. In the limit $s \rightarrow 0$, the unified $(q, s)$-entropy recovers the Renyi $q$-entropy

$$
R_{q}(X)=\frac{1}{1-q} \ln \left(\sum_{i=1}^{m} p_{X}(i)^{q}\right)
$$

Two specific entropic measures will be adopted through the paper. The first is the $(q, s)$-entropy exchange. For its description, we have to separate explicitly the principal system $Q$ from an imagined reference system $R$ and an environment $E$. The Hilbert spaces are denoted by $\mathcal{H}_{Q}, \mathcal{H}_{R}$, and $\mathcal{H}_{E}$, respectively. Under the action of quantum channel $\Phi^{Q}$, the initial state $\rho^{Q}$ of system $Q$ is mapped into $\Phi^{Q}\left(\rho^{Q}\right)$. To see the entanglement transmission, we consider a purification $\left|\psi^{Q R}\right\rangle \in \mathcal{H}_{Q} \otimes \mathcal{H}_{R}$, which is transformed into the final state

$$
\rho^{Q^{\prime} R^{\prime}}=\Phi^{Q} \otimes \mathrm{id}^{R}\left(\left|\psi^{Q R}\right\rangle\left\langle\psi^{Q R}\right|\right)
$$

of the system $Q R$. The system $R$ itself is not altered, i.e. $\operatorname{tr}_{Q}\left(\rho^{Q^{\prime} R^{\prime}}\right)=\operatorname{tr}_{Q}\left(\left|\psi^{Q R}\right\rangle\left\langle\psi^{Q R}\right|\right)$. Putting an environment $E$, we can reexpress the quantum channel $\Phi^{Q}$ in terms of unitary operator $\widetilde{U}$ on $\mathcal{H}_{E} \otimes \mathcal{H}_{Q}$ as

$$
\Phi^{Q}\left(\rho^{Q}\right)=\operatorname{tr}_{E}\left(\widetilde{\mathrm{U}}\left(\left|e_{0}\right\rangle\left\langle e_{0}\right| \otimes \rho^{Q}\right) \widetilde{\mathrm{U}}^{\dagger}\right)
$$

Since the final state $\left(\widetilde{\mathbf{U}} \otimes \mathbb{1}^{R}\right)\left|e_{0}\right\rangle \otimes\left|\psi^{Q R}\right\rangle$ of the triple system $E Q R$ is obviously pure, the final density operators $\rho^{E^{\prime}} \in \mathcal{L}_{+}\left(\mathcal{H}_{E}\right)$ and $\rho^{Q^{\prime} R^{\prime}} \in \mathcal{L}_{+}\left(\mathcal{H}_{Q} \otimes \mathcal{H}_{R}\right)$ have the same non-zero eigenvalues. We define the $(q, s)$-entropy exchange as

$$
\overline{\mathrm{H}}_{q}^{(s)}\left(\rho^{Q}, \Phi^{Q}\right):=\mathrm{H}_{q}^{(s)}\left(\rho^{Q^{\prime} R^{\prime}}\right)=\mathrm{H}_{q}^{(s)}\left(\rho^{E^{\prime}}\right)
$$

This quantity characterizes an amount of $(q, s)$-entropy introduced by the quantum channel $\Phi^{Q}$ into an initially pure environment $E$. The definition (2.12) is a direct extension of the Schumacher entropy exchange [6] to the considered entropic measure. It can be shown that the right-hand side of Eq. (2.12) depends only on the initial state $\rho^{Q}$ of the principal system and the quantum channel $\Phi^{Q}$ (for details, see subsection 12.4.1 in [1]). So further we can left out the superscript $Q$ of the principal system and merely write $\overline{\mathrm{H}}_{q}^{(s)}(\rho, \Phi)$.

Another specific entropic measure is the map $(q, s)$-entropy. It is defined within the Jamiołkowski-Choi representation of $\Phi[19,20]$. Let $\mathcal{H}_{Q}=\mathcal{H}_{R}=\mathcal{H}$ and $\{|\nu\rangle\}$ be an orthonormal basis in $\mathcal{H}$. To this basis we assign the normalized pure state

$$
\left|\phi_{+}\right\rangle:=\frac{1}{\sqrt{d}} \sum_{\nu=1}^{d}|\nu\rangle \otimes|\nu\rangle
$$

where $d$ is the dimensionality of $\mathcal{H}$. We then put the special operator

$$
\sigma(\Phi):=\Phi \otimes \operatorname{id}\left(\left|\phi_{+}\right\rangle\left\langle\phi_{+}\right|\right)
$$

acting on the doubled space $\mathcal{H}^{\otimes 2}$. The matrix $\mathrm{D}(\Phi)=d \sigma(\Phi)$ is called "dynamical matrix" or "Choi matrix" [20]. For each $X \in \mathcal{L}(\mathcal{H})$, the action of super-operator $\Phi$ can be recovered from $\mathrm{D}(\Phi)$ by means of the relation [1]

$$
\Phi(\mathrm{X})=\operatorname{tr}_{R}\left(\mathrm{D}(\Phi)\left(\mathbb{1} \otimes \mathrm{X}^{T}\right)\right)
$$

where $\mathrm{X}^{T}$ denotes the transpose operator to $\mathrm{X}$. The map $\Phi$ is completely positive, whenever the matrix $\mathrm{D}(\Phi)$ is positive. The condition $\operatorname{tr}_{R}(\mathrm{D}(\Phi))=\mathbb{1}$ is equivalent to that the map $\Phi$ is trace-preserving and the rescaled matrix $\sigma(\Phi)$ is of unit trace. For given channel $\Phi$, we define the map $(q, s)$-entropy by

$$
\mathrm{M}_{q}^{(s)}(\Phi):=\mathrm{H}_{q}^{(s)}(\sigma(\Phi))
$$

This is an extension of the standard map entropy introduced in [7] and further examined in [8]. The map entropy is used to characterize the decoherent behaviour of given channel. 


\section{EXTREMAL UNRAVELINGS OF A QUANTUM CHANNEL}

In this section, we study extremality of unravelings of a quantum channel with respect to unified entropies. Recall that representations of the form (2.3) are not unique [11]. For given map $\Phi$, there are many sets $\mathcal{A}=\left\{\mathrm{A}_{j}\right\}$ that enjoy Eq. (2.3). In the paper [21], each concrete set $\mathcal{A}=\left\{\mathrm{A}_{j}\right\}$ resulting in Eq. (2.3) is named an "unraveling" of the map $\Phi$. This terminology is due to Carmichael [22] who introduced this word for a representation of the master equation (for a review of this topic, see Ref. [23]). Following the method of Ref. [24], we introduce the matrix

$$
\Pi(\mathcal{A} \mid \rho):=\left[\left[\left\langle\mathrm{A}_{i} \sqrt{\rho}, \mathrm{A}_{j} \sqrt{\rho}\right\rangle_{\mathrm{hs}}\right]\right]=\left[\left[\operatorname{tr}\left(\mathrm{A}_{i}^{\dagger} \mathrm{A}_{j} \rho\right)\right]\right]
$$

for given density operator $\rho$ and channel unraveling $\mathcal{A}=\left\{\mathrm{A}_{i}\right\}$. The diagonal element $p_{i}=\operatorname{tr}\left(\mathrm{A}_{i}^{\dagger} \mathrm{A}_{i} \rho\right)$ is clearly positive and gives the $i$ th effect probability. Then the entropy $\mathrm{H}_{q}^{(s)}(\mathcal{A} \mid \rho)$ is defined by Eq. (2.8). It is well-known that two operator-sum representations of the same completely positive map are related as

$$
\mathrm{B}_{i}=\sum_{j} \mathrm{~A}_{j} u_{j i}
$$

where the matrix $\mathrm{U}=\left[\left[u_{i j}\right]\right]$ is unitary [1]. This statement can be obtained on the base of the ensemble classification theorem proved in Ref. [25]. If the two sets $\mathcal{A}=\left\{\mathrm{A}_{j}\right\}$ and $\mathcal{B}=\left\{\mathrm{B}_{i}\right\}$ fulfill Eq. (3.2), then we have

$$
\left\langle\mathrm{B}_{i} \sqrt{\rho}, \mathrm{B}_{k} \sqrt{\rho}\right\rangle_{\mathrm{hs}}=\sum_{j l} u_{j i}^{*} u_{l k}\left\langle\mathrm{~A}_{j} \sqrt{\rho}, \mathrm{A}_{l} \sqrt{\rho}\right\rangle_{\mathrm{hs}}
$$

or merely $\Pi(\mathcal{B} \mid \rho)=\mathrm{U}^{\dagger} \Pi(\mathcal{A} \mid \rho) \mathrm{U}$. In other words, the matrices $\Pi(\mathcal{A} \mid \rho)$ and $\Pi(\mathcal{B} \mid \rho)$ are unitarily similar $[24]$. By Hermiticity, all such matrices assigned to the same channel are unitarily similar to a unique (up to permutations) diagonal matrix $\Lambda=\operatorname{diag}\left(\lambda_{1}, \lambda_{2}, \ldots\right)$, where the $\lambda_{i}$ 's are the eigenvalues of each of these matrices. Hence any $\Pi(\mathcal{A} \mid \rho)$ is positive semidefinite. For given unraveling $\mathcal{A}=\left\{\mathrm{A}_{j}\right\}$, we obtain the concrete matrix $\Pi(\mathcal{A} \mid \rho)$ and $\operatorname{diagonalize}$ it through a unitary transformation $\mathrm{V}^{\dagger} \Pi(\mathcal{A} \mid \rho) \mathrm{V}=\Lambda$. Let us define a specific unraveling $\mathcal{A}_{\rho}^{(e x)}$ related to given $\mathcal{A}$ as

$$
\mathrm{A}_{i}^{(e x)}=\sum_{j} \mathrm{~A}_{j} v_{j i}
$$

where the unitary matrix $\mathrm{V}=\left[\left[v_{i j}\right]\right]$ diagonalizes $\Pi(\mathcal{A} \mid \rho)$. We shall show that the unraveling (3.4) enjoys the extremality property with respect to almost all the $(q, s)$-entropies.

Theorem III.1 Let $\rho$ be density operator on $\mathcal{H}$, and $\mathcal{A}$ an unraveling of quantum channel. For all $q>0$ and $s \neq 0$, there holds

$$
H_{q}^{(s)}\left(\mathcal{A}_{\rho}^{(e x)} \mid \rho\right) \leq H_{q}^{(s)}(\mathcal{A} \mid \rho)
$$

where the extremal unraveling $\mathcal{A}_{\rho}^{(e x)}$ is defined by Eq. 3.4.

Proof. We first suppose that $q \neq 1$. By construction, we have $\Pi\left(\mathcal{A}_{\rho}^{(e x)} \mid \rho\right)=\Lambda$ with the probabilities $\lambda_{j}$ of effects. Due to $\Pi(\mathcal{A} \mid \rho)=\mathrm{V} \wedge \mathrm{V}^{\dagger}$, the probabilities $p_{i}=\operatorname{tr}\left(\mathrm{A}_{i}^{\dagger} \mathrm{A}_{i} \rho\right)$ of different effects of $\mathcal{A}$ is related to the $\lambda_{j}$ 's by

$$
p_{i}=\sum_{j} v_{i j} \lambda_{j} v_{i j}^{*}=\sum_{j} w_{i j} \lambda_{j}
$$

Here numbers $w_{i j}=v_{i j} v_{i j}^{*}$ are elements of unistochastic matrix, whence $\sum_{i} w_{i j}=1$ for all $j$ and $\sum_{j} w_{i j}=1$ for all $i$. The function $x \mapsto x^{q}$ is concave for $0<q<1$ and convex for $1<q$. Applying Jensen's inequality to this function, we obtain

$$
\sum_{i} p_{i}^{q}=\sum_{i}\left(\sum_{j} w_{i j} \lambda_{j}\right)^{q}\left\{\begin{array}{cc}
\geq, & 0<q<1 \\
\leq, & 1<q
\end{array}\right\} \quad \sum_{i} \sum_{j} w_{i j} \lambda_{j}^{q}=\sum_{j} \lambda_{j}^{q}
$$

in view of the above unistochasticity. The function $y \mapsto y^{s} / s$ monotonically increases for all $s \neq 0$, whence

$$
\frac{1}{s}\left(\sum_{i} p_{i}^{q}\right)^{s}\left\{\begin{array}{cc}
\geq, & 0<q<1 \\
\leq, & 1<q
\end{array}\right\} \frac{1}{s}\left(\sum_{j} \lambda_{j}^{q}\right)^{s}
$$

Since the term $(1-q)$ is positive for $q<1$ and negative for $1<q$, the relations (3.8) are merely combined as

$$
\frac{1}{(1-q) s}\left(\sum_{i} p_{i}^{q}\right)^{s} \geq \frac{1}{(1-q) s}\left(\sum_{j} \lambda_{j}^{q}\right)^{s} \text {. }
$$


Due to the definition (2.8), the inequality (3.9) provides (3.5). In the case $q=1$, we deal with the Shannon entropy. Applying Jensen's inequality to the concave function $x \mapsto-x \ln x$ completes the proof of this case.

For prescribed state $\rho$ and given unraveling of quantum channel all the unified $(q, s)$-entropies with parameter $s \neq 0$ are minimized by the extremal unraveling, which is built in line with Eq. (3.4). For the Shannon entropy, a question with "minimal" unraveling was considered in Ref. [26] and later in Ref. [21]. It was noted in Ref. [24] that diagonalizing the matrix $\Pi(\mathcal{A} \mid \rho)$ is formally equivalent to the extreme condition of Ref. [21]. The latter condition has been obtained by calculation with Lagrange's multipliers which is rather local in spirit. These reasons are complemented by the above proof based purely on the concavity property. As it was shown in Ref. [24], the Rényi entropies enjoy the extremality property with unraveling (3.4) only for order $q \in(0 ; 1)$. In this regard, the Rényi entropies differ from other considered entropies.

A quantum state is characterized by the probabilities of the outcomes of every conceivable test [27]. So the measurements are quantum operations of special conceptual interest. A general measurement is described by the set $\left\{\mathrm{M}_{i}\right\}$ of measurement operators [1]. Suppose that $\rho$ is density operator of the system right before the measurement. Separate terms of the sum

$$
\sum_{i} \mathrm{M}_{i} \rho \mathrm{M}_{i}^{\dagger}
$$

are related to different outcomes of the measurement. Since the probabilities $\operatorname{tr}\left(\mathrm{M}_{i}^{\dagger} \mathrm{M}_{i} \rho\right)$ are summed to one, the measurement operators enjoy the equality in Eq. (2.4). A standard measurement is described by the set $\left\{\mathrm{P}_{j}\right\}$ of mutually orthogonal projectors. As a rule, projective measurements are easier to realize experimentally. One of basic properties of the von Neumann entropy is that it cannot be decreased by a projective measurement (see, e.g., theorem 11.9 in [1]). For given measurement $\left\{\mathrm{P}_{j}\right\}$, the output density operator is expressed as [1]

$$
\mathcal{C}(\rho)=\sum_{j} \mathrm{P}_{j} \rho \mathrm{P}_{j}
$$

In matrix analysis, an operation of such a kind is referred to as "pinching" (for details, see section IV.2 of Ref. [12]). The non-decreasing of the von Neumann entropy is posed as $\mathrm{H}_{1}(\rho) \leq \mathrm{H}_{1}(\mathcal{C}(\rho))$. As it is shown in Ref. [4], all the unified entropies are non-decreasing under projective measurements, namely

$$
\mathrm{H}_{q}^{(s)}(\rho) \leq \mathrm{H}_{q}^{(s)}(\mathcal{C}(\rho))
$$

Hence we can see that $\mathrm{H}_{q}^{(s)}(\rho) \leq H_{q}^{(s)}\left(\mathcal{C}_{\rho}^{(e x)} \mid \rho\right)$, where $\mathcal{C}_{\rho}^{(e x)}$ denotes extremal unraveling of the projective measurement. In other words, the entropy $\mathrm{H}_{q}^{(s)}(\rho)$ is a lower bound for the extremal unraveling entropy $H_{q}^{(s)}\left(\mathcal{C}_{\rho}^{(e x)} \mid \rho\right)$. The latter inequality is always saturated, when the measurement is carried out in the basis of the eigenstates of $\rho$. We shall extend this treatment to other quantum channels. Such an extension takes place, when the Kraus operators of extremal unraveling fulfill a certain condition.

Theorem III.2 Suppose that the Kraus operators $\mathrm{A}_{i}^{(e x)}$ of extremal unraveling of a quantum channel satisfy

$$
\operatorname{tr}\left(\mathrm{A}_{i}^{(e x) \dagger} \mathrm{A}_{i}^{(e x)}\right)=1
$$

for all values of index $i$. For $0<q$ and $s \neq 0$ as well as for $0<q<1$ and $s=0$, we then have

$$
\mathrm{H}_{q}^{(s)}(\rho) \leq H_{q}^{(s)}\left(\mathcal{A}_{\rho}^{(e x)} \mid \rho\right)
$$

Proof. We first suppose that $q \neq 1$. By definition, the matrix $\Pi\left(\mathcal{A}_{\rho}^{(e x)} \mid \rho\right)$ is diagonal. Calculating the trace in the eigenbasis $\{|j\rangle\}$ of $\rho$, we rewrite diagonal elements as

$$
\lambda_{i}=\operatorname{tr}\left(\mathrm{A}_{i}^{(e x) \dagger} \mathrm{A}_{i}^{(e x)} \rho\right)=\sum_{j} \varsigma_{j}\left\langle j\left|\mathrm{~A}_{i}^{(e x) \dagger} \mathrm{A}_{i}^{(e x)}\right| j\right\rangle
$$

where the $\varsigma_{j}$ 's are eigenvalues of $\rho$. The numbers $t_{i j}=\left\langle j\left|\mathrm{~A}_{i}^{(e x) \dagger} \mathrm{A}_{i}^{(e x)}\right| j\right\rangle$ form a double-stochastic matrix due to

$$
\sum_{j} t_{i j}=\operatorname{tr}\left(\mathrm{A}_{i}^{(e x) \dagger} \mathrm{A}_{i}^{(e x)}\right)=1, \quad \sum_{i} t_{i j}=\langle j \mid j\rangle=1 .
$$

Here the first follows from Eq. (3.13), the second follows from the equality in Eq. (2.4). Combining both the relations of Eq. 3.16 with the Jensen inequality, we obtain

$$
\sum_{i} \lambda_{i}^{q}=\sum_{i}\left(\sum_{j} t_{i j} \varsigma_{j}\right)^{q}\left\{\begin{array}{cc}
\geq, & 0<q<1 \\
\leq, & 1<q
\end{array}\right\} \quad \sum_{i} \sum_{j} t_{i j} \varsigma_{j}^{q}=\sum_{j} \varsigma_{j}^{q} .
$$


As the function $y \mapsto y^{s} / s$ monotonically increases for $s \neq 0$, these inequalities lead to

$$
\frac{1}{s}\left(\sum_{i} \lambda_{i}^{q}\right)^{s}\left\{\begin{array}{lc}
\geq, & 0<q<1 \\
\leq, & 1<q
\end{array}\right\} \frac{1}{s}\left(\sum_{j} \varsigma_{j}^{q}\right)^{s}
$$

Multiplying Eq. (3.18) by the factor $(1-q)^{-1}$, which is positive for $0<q<1$ and negative for $1<q$, we complete the proof for $q \neq 1$ and $s \neq 0$. In the case $q=1$, when the von Neumann and Shannon entropies are dealt, we merely combine Eq. (3.16) with the concavity of the function $x \mapsto-x \ln x$. When $s=0$, we apply the relations

$$
\mathrm{R}_{q}(\rho)=(1-q)^{-1} \ln \left(1+(1-q) \mathrm{T}_{q}(\rho)\right), \quad R_{q}\left(\mathcal{A}_{\rho}^{(e x)} \mid \rho\right)=(1-q)^{-1} \ln \left(1+(1-q) T_{q}\left(\mathcal{A}_{\rho}^{(e x)} \mid \rho\right)\right)
$$

which at once follow from the definitions of the Rényi and Tsallis $q$-entropies. Since the inequality (3.14) holds for the Tsallis case $(s=1)$ and the function $y \mapsto(1-q)^{-1} \ln (1+(1-q) y)$ is increasing for $q<1$, the Rényi $q$-entropies also enjoy Eq. (3.14) for $0<q<1$.

Thus, if the condition (3.13) holds then the extremal unraveling entropy $H_{q}^{(s)}\left(\mathcal{A}_{\rho}^{(e x)} \mid \rho\right)$ is bounded from below by the entropy of the input state $\mathrm{H}_{q}^{(s)}(\rho)$. This property takes place for all unified $(q, s)$-entropies, except for the Rényi $q$-entropies of order $q>1$. Here we again see some distinctions of the Rényi entropies from the rest entropies considered. The inequality (3.14) may also be regarded as an estimate on the entropy of input state of a channel, when the extremal unraveling entropy is known (exactly or approximately) from other reasons.

\section{CONTINUITY ESTIMATES ON THE MAP $(q, s)$-ENTROPIES}

One of essential properties of the von Neumann entropy is its continuity firstly stated by Fannes [28]. Fannes' inequality has been generalized to the Tsallis entropy [29, 30] and its partial sums [31]. Continuity estimates of Fannes type have also been derived for the quantum conditional entropy [32] as well as for the standard quantum relative entropy [33] and its $q$-extension [34]. Below we will consider inequalities of Fannes type for the map $(q, s)$ entropies. Continuity estimates on the unified $(q, s)$-entropies were derived in Ref. [4]. They are based on the known inequalities with the Tsallis entropies [29, 30]. Combining the estimates of Ref. [4] with the definition (2.16), we obtain the following. Let $\Phi, \Psi$ be quantum channels on $d$-dimensional states. For the parameter range

$$
\{(q, s): 0<q<1, s \in(-\infty ;-1] \cup[0 ;+1]\}
$$

under the condition $\|\sigma(\Phi)-\sigma(\Psi)\|_{1}=2 t \leq q^{1 /(1-q)}$, there holds

$$
\left|\mathrm{M}_{q}^{(s)}(\Phi)-\mathrm{M}_{q}^{(s)}(\Psi)\right| \leq(2 t)^{q} \ln _{q} d+\eta_{q}(2 t) .
$$

Define the factor $\varkappa_{s}=d^{2(q-1)}$ for $s \in[-1 ; 0]$ and $\varkappa_{s}=1$ for $s \in[+1 ;+\infty)$. For the parameter range

$$
\{(q, s): 1<q, s \in[-1 ; 0] \cup[+1 ;+\infty)\}
$$

under the condition $(1 / 2)\|\sigma(\Phi)-\sigma(\Psi)\|_{1}=t \leq(d-1) / d$, there holds

$$
\left|\mathrm{M}_{q}^{(s)}(\Phi)-\mathrm{M}_{q}^{(s)}(\Psi)\right| \leq \varkappa_{s}\left[t^{q} \ln _{q}(d-1)+T_{q}(t, 1-t)\right] .
$$

Here $T_{q}(t, 1-t)$ is the binary Tsallis entropy. The validity ranges $0 \leq 2 t \leq q^{1 /(1-q)}$ for (4.2) and $0 \leq t \leq(d-1) / d$ for (4.4) are essential and obtained as intervals of non-decreasing of the corresponding right-hand sides.

In principle, the continuity property is established by means of Eqs. (4.2) and (4.4). At the same time, the norm $\|\sigma(\Phi)-\sigma(\Psi)\|_{1}$ is sufficiently difficult for calculation in general form. The Frobenius norm distance $\|\sigma(\Phi)-\sigma(\Psi)\|_{2}$ is easier to estimate. By Eq. (2.14), there holds

$$
|\sigma(\Phi)-\sigma(\Psi)|^{2}=\frac{1}{d^{2}} \sum_{\mu \nu \xi}\{\Phi(|\mu\rangle\langle\nu|)-\Psi(|\mu\rangle\langle\nu|)\}\{\Phi(|\nu\rangle\langle\xi|)-\Psi(|\nu\rangle\langle\xi|)\} \otimes|\mu\rangle\langle\xi| .
$$

Hence, in view of $\operatorname{tr}(\mathrm{X} \otimes \mathrm{Y})=\operatorname{tr}(\mathrm{X}) \operatorname{tr}(\mathrm{Y})$ and the linearity of the trace, we obtain

$$
\operatorname{tr}\left(|\sigma(\Phi)-\sigma(\Psi)|^{2}\right)=\frac{1}{d^{2}} \sum_{\mu \nu} \operatorname{tr}\left(|\Phi(|\mu\rangle\langle\nu|)-\Psi(|\mu\rangle\langle\nu|)|^{2}\right)=\frac{1}{d^{2}} \sum_{\mu \nu}\|\Phi(|\mu\rangle\langle\nu|)-\Psi(|\mu\rangle\langle\nu|)\|_{2}^{2} .
$$


In other words, the Frobenius norm distance $\|\sigma(\Phi)-\sigma(\Psi)\|_{2}$ is expressed as the 2-mean

$$
\|\sigma(\Phi)-\sigma(\Psi)\|_{2}=\mathfrak{M}_{2}\left(\|(\Phi-\Psi)(|\mu\rangle\langle\nu|)\|_{2}\right)
$$

of the particular distances $\|(\Phi-\Psi)(|\mu\rangle\langle\nu|)\|_{2}$ taken with equal weights. Other questions, in which the Frobenius norm distance is very useful, are treated in Ref. [16]. So, it is of interest to pose continuity estimates on the map entropies in terms of the distance (4.7). On the other hand, the inequalities of Fannes type are naturally formulated in terms of the trace norm distance. Hence we are interested in relations between the trace and Frobenius norms. This issue is considered in Appendix A Taking $q=2$ in (A5), we obtain $\|\mathrm{X}\|_{1} \leq \sqrt{d}\|\mathrm{X}\|_{2}$ for each $\mathrm{X} \in \mathcal{L}(\mathcal{H})$.

In the intervals of non-decreasing right-hand sides, the upper bounds (4.2) and (4.4) are recast with larger $\sqrt{d}\|\sigma(\Phi)-\sigma(\Psi)\|_{2}$ instead of $\|\sigma(\Phi)-\sigma(\Psi)\|_{1}$ as follows. For the parameter range (4.1), there holds

$$
\left|\mathrm{M}_{q}^{(s)}(\Phi)-\mathrm{M}_{q}^{(s)}(\Psi)\right| \leq d^{q / 2}(2 \tau)^{q} \ln _{q} d+\eta_{q}(\sqrt{d} 2 \tau)
$$

provided that $\|\sigma(\Phi)-\sigma(\Psi)\|_{2}=2 \tau \leq q^{1 /(1-q)} d^{-1 / 2}$. For the parameter range (4.3), there holds

$$
\left|\mathrm{M}_{q}^{(s)}(\Phi)-\mathrm{M}_{q}^{(s)}(\Psi)\right| \leq \varkappa_{s}\left[d^{q / 2} \tau^{q} \ln _{q}(d-1)+T_{q}(\sqrt{d} \tau, 1-\sqrt{d} \tau)\right]
$$

provided that $(1 / 2)\|\sigma(\Phi)-\sigma(\Psi)\|_{2}=\tau \leq(d-1) d^{-3 / 2}$. So, we have arrived at upper bounds in terms of the Frobenius norm distance between the two rescaled dynamical matrices. However, the validity ranges of Eqs. (4.8) and (4.9), particularly the former, seem to be too restrictive for sufficiently large $d$. Since the operator $\sigma(\Phi)-\sigma(\Psi)$ is traceless Hermitian and $\operatorname{tr}(\sigma(\Phi))=\operatorname{tr}(\sigma(\Psi))=1$, the trace and Frobenius norms obey $\|\sigma(\Phi)-\sigma(\Psi)\|_{1} \leq 2$ and $\|\sigma(\Phi)-\sigma(\Psi)\|_{2} \leq \sqrt{2}$, respectively. Below, we will derive upper bounds that hold for all $\|\sigma(\Phi)-\sigma(\Psi)\|_{2} \leq \sqrt{2}$.

Due to Csiszár, the classical Fano inequality leads to an improvement of the original Fannes' bound (see theorem 3.8 and its proof in Ref. [35]). Developing this point was for the Tsallis entropies [36], we obtain the inequality

$$
\left|\mathrm{T}_{q}(\rho)-\mathrm{T}_{q}(\omega)\right| \leq t^{q} \ln _{q}[d(d-1)]+\frac{t^{q}-q t}{1-q}=\frac{(d(d-1))^{1-q} t^{q}-q t}{1-q}
$$

for $0<q<1$ and all $(1 / 2)\|\rho-\omega\|_{1}=t \leq 1$, and the inequality

$$
\left|\mathrm{T}_{q}(\rho)-\mathrm{T}_{q}(\sigma)\right| \leq t^{q} \ln _{q}(d-1)+T_{q}(t, 1-t)
$$

for $1<q$ and $(1 / 2)\|\rho-\omega\|_{1}=t \leq(d-1) / d$. The second is exactly those bound that was derived immediately in Ref. [30]. In comparison with Eq. (4.2), the upper bound (4.10) is valid for all acceptable values of the norm $\|\rho-\omega\|_{1}$, including its maximal value 2. We shall now modify Eq. (4.11) in this regard. The bound (4.11) was derived in Ref. [36] with use of the relation

$$
T_{q}(X)-T_{q}(Y) \leq T_{q}(X \mid Y) \leq P_{e}^{q} \ln _{q}(m-1)+T_{q}\left(P_{e}, 1-P_{e}\right)
$$

Here random variables $X$ and $Y$ take the same $m$ possible values, $T_{q}(X \mid Y)$ is the conditional $q$-entropy, and $P_{e}$ is the probability of error, i.e. the probability of that $X \neq Y$. The right-hand side of Eq. (4.12) is an extension of the classical Fano inequality to the conditional Tsallis entropy (for its properties, see Ref. [37]). We rewrite the right-hand side of Eq. (4.12) in the form

$$
\frac{1-\left(1-P_{e}\right)^{q}-(m-1)^{1-q} P_{e}^{q}}{q-1} \leq \frac{q P_{e}-(m-1)^{1-q} P_{e}^{q}}{q-1}
$$

which follows from the inequality $1-\left(1-P_{e}\right)^{q}=\int_{0}^{P_{e}} q(1-x)^{q-1} d x \leq \int_{0}^{P_{e}} q d x=q P_{e}$ in view of $q>1$. As function of $P_{e}$, the left-hand side of Eq. (4.13) monotonically increases up to the point $P_{e}=(m-1) / m$, where the derivative is zero. But the derivative of right-hand side of Eq. (4.13) vanishes at the point $P_{e}=m-1$. So the interval of nondecreasing becomes wider, though the bound with the right-hand side of Eq. (4.13) will somewhat weaker. Setting $m=d$, we consider the probabilities of $X$ and $Y$ as eigenvalues of $\rho$ and $\omega$, respectively. The joint probability mass function of $X$ and $Y$ can be built in such a way that $P_{e}=(1 / 2) \sum_{i}\left|p_{X}(i)-p_{Y}(i)\right| \leq(1 / 2)\|\rho-\omega\|_{1}($ the equality on the left is a part of the coupling inequality [38], the inequality on the right follows from lemma 11.1 of Ref. [35]). Combining this with Eq. (4.12) leads to the inequality of Fannes type in the form

$$
\left|\mathrm{T}_{q}(\rho)-\mathrm{T}_{q}(\omega)\right| \leq \frac{q t-(d-1)^{1-q} t^{q}}{q-1}
$$


where $t=(1 / 2)\|\rho-\omega\|_{1}$ may take all possible values from the range $0 \leq t \leq 1$. Replacing $t$ with larger $\sqrt{d} \tau$, we get the upper bound

$$
\left|\mathrm{T}_{q}(\rho)-\mathrm{T}_{q}(\omega)\right| \leq \frac{q \sqrt{d} \tau-(d-1)^{1-q} d^{q / 2} \tau^{q}}{q-1}
$$

where $\tau=(1 / 2)\|\rho-\omega\|_{2}$. The above replacing is valid under the constraint $\sqrt{d} \tau \leq d-1$, i.e. $\|\rho-\omega\|_{2} \leq 2(d-1) / \sqrt{d}$. The latter always holds, since $\|\rho-\omega\|_{2} \leq \sqrt{2}$ for two density matrices and $\sqrt{d / 2} \leq d-1$ for all $d \geq 2$. In other words, the upper bound (4.15) is dealt for all possible values of the Frobenius norm distance between states (and $1<q$ ). In the same manner, from Eq. (4.10) we obtain

$$
\left|\mathrm{T}_{q}(\rho)-\mathrm{T}_{q}(\omega)\right| \leq \frac{d^{1-q / 2}(d-1)^{1-q} \tau^{q}-q \sqrt{d} \tau}{1-q} .
$$

The right-hand side of Eq. (4.10) monotonically increases up to the point $t=d(d-1)$, whence the replacing is formally valid under the constraint $\sqrt{d} \tau \leq d(d-1)$, i.e $\|\rho-\omega\|_{2} \leq 2 \sqrt{d}(d-1)$. So the upper bound (4.16) is also dealt for all possible values of the Frobenius norm distance between states (and $0<q<1$ ). Repeating the reasons of section 3 of Ref. [4] with new bounds on the Tsallis entropy, we obtain the following statement.

Theorem IV.1 Let $\rho$ and $\omega$ be density operators on d-dimensional Hilbert space $\mathcal{H}$. For the parameter range 4.1 and all possible values of the distance $\tau=(1 / 2)\|\rho-\omega\|_{2}$, there holds

$$
\left|\mathrm{H}_{q}^{(s)}(\rho)-\mathrm{H}_{q}^{(s)}(\omega)\right| \leq \frac{d^{1-q / 2}(d-1)^{1-q} \tau^{q}-q \sqrt{d} \tau}{1-q} .
$$

For the parameter range (4.3) and all possible values of the distance $\tau=(1 / 2)\|\rho-\omega\|_{2}$, there holds

$$
\left|\mathrm{H}_{q}^{(s)}(\rho)-\mathrm{H}_{q}^{(s)}(\omega)\right| \leq \varkappa_{s} \frac{q \sqrt{d} \tau-(d-1)^{1-q} d^{q / 2} \tau^{q}}{q-1}
$$

where the factor $\varkappa_{s}=d^{2(q-1)}$ for $s \in[-1 ; 0]$ and $\varkappa_{s}=1$ for $s \in[+1 ;+\infty)$.

Hence we obtain another upper bounds on the map $(q, s)$-entropies in terms of the quantity (4.7). Designating $\|\sigma(\Phi)-\sigma(\Psi)\|_{2}=2 \tau$, we have

$$
\begin{aligned}
& \left|\mathrm{M}_{q}^{(s)}(\Phi)-\mathrm{M}_{q}^{(s)}(\Psi)\right| \leq \frac{d^{1-q / 2}(d-1)^{1-q} \tau^{q}-q \sqrt{d} \tau}{1-q} \\
& \left|\mathrm{M}_{q}^{(s)}(\Phi)-\mathrm{M}_{q}^{(s)}(\Psi)\right| \leq \varkappa_{s} \frac{q \sqrt{d} \tau-(d-1)^{1-q} d^{q / 2} \tau^{q}}{q-1}
\end{aligned}
$$

in the parameter ranges (4.1) and (4.3), respectively. In comparison with Eqs. (4.8) and (4.9), these bounds are somewhat weaker, but their validity intervals for $\|\sigma(\Phi)-\sigma(\Psi)\|_{2}$ do not depend on the dimensionality of the Hilbert space. However, for sufficiently small values of this distance we prefer the bounds (4.8) and (4.9).

\section{SOME ADDITIVITY PROPERTIES OF THE MAP $(q, s)$-ENTROPIES}

In this section, we examine properties of the map $(q, s)$-entropies with respect to tensor product of a pair of quantum channels. Broad use of the Tsallis entropy in non-extensive statistical mechanics stems from the fact that it does not share the additivity in the following sense [39]. If two random variable $X$ and $Y$ are independent then the Shannon entropy of the joint distribution $H_{1}(X, Y)=H_{1}(X)+H_{1}(Y)$. In the quantum regime, the von Neumann entropy enjoys $\mathrm{H}_{1}\left(\rho^{Q} \otimes \rho^{R}\right)=\mathrm{H}_{1}\left(\rho^{Q}\right)+\mathrm{H}_{1}\left(\rho^{R}\right)$. In general, we have the subadditivity [2]

$$
\mathrm{H}_{1}\left(\rho^{Q R}\right) \leq \mathrm{H}_{1}\left(\rho^{Q}\right)+\mathrm{H}_{1}\left(\rho^{R}\right)
$$

where reduced densities $\rho^{Q}$ and $\rho^{R}$ are obtained from $\rho^{Q R}$ by taking the partial trace. The equality in Eq. (5.1) takes place only for the above case of product states. The Tsallis entropies do not enjoy the additivity with the product states, though the subadditivity of quantum $q$-entropy is still obeyed for $q>1$, namely

$$
\mathrm{H}_{q}\left(\rho^{Q R}\right) \leq \mathrm{H}_{q}\left(\rho^{Q}\right)+\mathrm{H}_{q}\left(\rho^{R}\right) .
$$


This fact has been conjectured in Ref. [40] and later proved in Ref. [41]. Concerning additivity properties, the unified entropies succeed to the Tsallis entropies [3]. In particular, we have the subadditivity of the quantum $(q, s)$-entropy for $q>1$ and $s>1 / q[4]$. Additivity properties of the map $(q, s)$-entropies with respect to the tensor product of quantum channels are posed as follows.

Theorem V.1 Let $\Phi_{1}$ and $\Phi_{2}$ be quantum channels. For $q>0$ and all real $s$, the map $(q, s)$-entropy satisfies

$$
\mathrm{M}_{q}^{(s)}\left(\Phi_{1} \otimes \Phi_{2}\right)=\mathrm{M}_{q}^{(s)}\left(\Phi_{1}\right)+\mathrm{M}_{q}^{(s)}\left(\Phi_{2}\right)+(1-q) s \mathrm{M}_{q}^{(s)}\left(\Phi_{1}\right) \mathrm{M}_{q}^{(s)}\left(\Phi_{2}\right) .
$$

Proof. The claim is based on the two point. The first is the expression for unified $(q, s)$-entropy of a product state, which is formulated as [3]

$$
\mathrm{H}_{q}^{(s)}(\rho \otimes \omega)=\mathrm{H}_{q}^{(s)}(\rho)+\mathrm{H}_{q}^{(s)}(\omega)+(1-q) s \mathrm{H}_{q}^{(s)}(\rho) \mathrm{H}_{q}^{(s)}(\omega) .
$$

The second is the important result that the dynamical matrix $\mathrm{D}\left(\Phi_{1} \otimes \Phi_{2}\right)$ is unitarily similar to the product matrix $\mathrm{D}\left(\Phi_{1}\right) \otimes \mathrm{D}\left(\Phi_{2}\right)[\underline{8}]$, whence

$$
\mathrm{M}_{q}^{(s)}\left(\Phi_{1} \otimes \Phi_{2}\right)=\mathrm{H}_{q}^{(s)}\left(\sigma\left(\Phi_{1}\right) \otimes \sigma\left(\Phi_{2}\right)\right) .
$$

Combining Eqs. (5.4) and (5.5) finally leads to Eq. (5.3).

For the case $s=0$, the relation (5.3) gives the additivity of the Rényi map entropies. The latter has been proved in Ref. [8]. It is known that the rank of the dynamical matrix is equal to the minimal number of terms needed in the operator-sum representation [7]. So if one of channels $\Phi_{1}$ and $\Phi_{2}$ represents a unitary evolution, then its dynamical matrix is of rank one and its map $(q, s)$-entropy is zero. Then the relation (5.3) claims the additivity of the map $(q, s)$-entropy. If no one of $\Phi_{1}$ and $\Phi_{2}$ represents a unitary evolution, then both the dynamical matrices are of rank $\geq 2$ and both the map entropies are non-zero. In this case, the map $(q, s)$-entropy is strictly subadditive for $0<q<1$ and $s<0$ as well as for $1<q$ and $0<s$, i.e.

$$
\mathrm{M}_{q}^{(s)}\left(\Phi_{1} \otimes \Phi_{2}\right)<\mathrm{M}_{q}^{(s)}\left(\Phi_{1}\right)+\mathrm{M}_{q}^{(s)}\left(\Phi_{2}\right), \quad\{0<q<1, s<0\} \bigcup\{1<q, 0<s\} .
$$

Further, the map $(q, s)$-entropy is strictly superadditive for $0<q<1$ and $0<s$ as well as for $1<q$ and $s<0$, i.e.

$$
\mathrm{M}_{q}^{(s)}\left(\Phi_{1} \otimes \Phi_{2}\right)>\mathrm{M}_{q}^{(s)}\left(\Phi_{1}\right)+\mathrm{M}_{q}^{(s)}\left(\Phi_{2}\right), \quad\{0<q<1,0<s\} \bigcup\{1<q, s<0\} .
$$

Using the map $(q, s)$-entropies, we can herewith separate quantum channels that represent a unitary evolution. On the contrary, the map Rényi entropies are additive irrespectively to channel features [8].

We shall now extend the inequality of Lindblad [42] with the von Neumann entropy exchange to a certain subclass of unified entropies. Hence, estimating of the output entropy of maximally entangled input state will be carried out. This question is arisen within studies of so-called "additivity conjecture" concerning a product quantum channel [8]. An extension of the Lindblad inequality is posed as follows.

Theorem V.2 Let $\rho$ be density operator, and $\Phi$ a quantum channel. For $q>1$ and $s \geq q^{-1}$, the inequality

$$
\left|\mathrm{H}_{q}^{(s)}(\rho)-\overline{\mathrm{H}}_{q}^{(s)}(\rho, \Phi)\right| \leq \mathrm{H}_{q}^{(s)}(\Phi(\rho)) \leq \mathrm{H}_{q}^{(s)}(\rho)+\overline{\mathrm{H}}_{q}^{(s)}(\rho, \Phi),
$$

including permutations of the three entropies, takes place.

Proof. Within the proof, we recall meaning of the systems $E, Q, R$ from Eqs. (2.10)-(2.12) and the respective notation. Using one result of Ref. [41], both the subadditivity and triangle inequality have been stated for the quantum $(q, s)$-entropies for $q>1, s \geq q^{-1}$ [4]. For the reduced densities

$$
\rho^{Q^{\prime}}=\operatorname{tr}_{E}\left(\rho^{E^{\prime} Q^{\prime}}\right), \quad \rho^{E^{\prime}}=\operatorname{tr}_{Q}\left(\rho^{E^{\prime} Q^{\prime}}\right)
$$

obtained from the output density operator $\rho^{E^{\prime} Q^{\prime}}$, and the parameter values $q>1, s \geq q^{-1}$, we have the relations

$$
\left|\mathrm{H}_{q}^{(s)}\left(\rho^{E^{\prime}}\right)-\mathrm{H}_{q}^{(s)}\left(\rho^{Q^{\prime}}\right)\right| \leq \mathrm{H}_{q}^{(s)}\left(\rho^{E^{\prime} Q^{\prime}}\right) \leq \mathrm{H}_{q}^{(s)}\left(\rho^{E^{\prime}}\right)+\mathrm{H}_{q}^{(s)}\left(\rho^{Q^{\prime}}\right) .
$$

The inequality on the left is the triangle inequality, the inequality on the right expresses the subadditivity. We then rewrite (5.10) as

$$
\left|\overline{\mathrm{H}}_{q}^{(s)}\left(\rho^{Q}, \Phi^{Q}\right)-\mathrm{H}_{q}^{(s)}\left(\Phi^{Q}\left(\rho^{Q}\right)\right)\right| \leq \mathrm{H}_{q}^{(s)}\left(\rho^{Q}\right) \leq \overline{\mathrm{H}}_{q}^{(s)}\left(\rho^{Q}, \Phi^{Q}\right)+\mathrm{H}_{q}^{(s)}\left(\Phi^{Q}\left(\rho^{Q}\right)\right),
$$


in view of the definition (2.12) and $\rho^{Q^{\prime}}=\Phi^{Q}\left(\rho^{Q}\right)$. It was also used that the systems $Q, R$ initially share the same pure state and the system $R$ itself is not altered. By doing some simple algebra, we further obtain

$$
\left|\mathrm{H}_{q}^{(s)}\left(\Phi^{Q}\left(\rho^{Q}\right)\right)-\mathrm{H}_{q}^{(s)}\left(\rho^{Q}\right)\right| \leq \overline{\mathrm{H}}_{q}^{(s)}\left(\rho^{Q}, \Phi^{Q}\right) \leq \mathrm{H}_{q}^{(s)}\left(\Phi^{Q}\left(\rho^{Q}\right)\right)+\mathrm{H}_{q}^{(s)}\left(\rho^{Q}\right),
$$

and the inequality (5.8) (in which the superscript $Q$ is already left out).

For the von Neumann entropy, an analog of the inequality (5.8) plus permutations was presented by Lindblad [42]. The writers of Ref. [8] used Lindblad's inequality for estimation of the entropy of an output state arising from a maximally entangled input state. We shall develop this issue with respect to the unified $(q, s)$-entropies.

Theorem V.3 Let $\Phi_{1}$ and $\Phi_{2}$ be quantum channels on $\mathcal{H}$, and $\left|\psi_{+}\right\rangle \in \mathcal{H}^{\otimes 2}$ a maximally entangled state. For $q>1$ and $s \geq q^{-1}$, there holds

$$
\left|\mathrm{M}_{q}^{(s)}\left(\Phi_{1}\right)-\mathrm{M}_{q}^{(s)}\left(\Phi_{2}\right)\right| \leq \mathrm{H}_{q}^{(s)}\left(\Phi_{1} \otimes \Phi_{2}\left(\left|\psi_{+}\right\rangle\left\langle\psi_{+}\right|\right)\right) \leq \mathrm{M}_{q}^{(s)}\left(\Phi_{1}\right)+\mathrm{M}_{q}^{(s)}\left(\Phi_{2}\right) .
$$

Proof. Following the method of Ref. [8], we represent the output density operator on the doubled space as

$$
\Phi_{1} \otimes \Phi_{2}\left(\left|\psi_{+}\right\rangle\left\langle\psi_{+}\right|\right)=\left(\Phi_{1} \otimes \mathrm{id}\right) \circ\left(\mathrm{id} \otimes \Phi_{2}\right)\left(\left|\psi_{+}\right\rangle\left\langle\psi_{+}\right|\right)=\Phi_{1} \otimes \mathrm{id}\left(\sigma\left(\Phi_{2}\right)\right) .
$$

It is easy to check that tracing-out the second space from the density matrix $\sigma\left(\Phi_{2}\right)=\mathrm{id} \otimes \Phi_{2}\left(\left|\psi_{+}\right\rangle\left\langle\psi_{+}\right|\right)$gives the maximally mixed state $\rho_{*}=\mathbb{1} / d$ on $\mathcal{H}$. Applying Eq. (5.8) to the density $\sigma\left(\Phi_{2}\right)$ and the channel $\Phi_{1} \otimes$ id, we then obtain

$$
\left|\mathrm{M}_{q}^{(s)}\left(\Phi_{2}\right)-\overline{\mathrm{H}}_{q}^{(s)}\left(\rho_{*}, \Phi_{1}\right)\right| \leq \mathrm{H}_{q}^{(s)}\left(\Phi_{1} \otimes \Phi_{2}\left(\left|\psi_{+}\right\rangle\left\langle\psi_{+}\right|\right)\right) \leq \mathrm{M}_{q}^{(s)}\left(\Phi_{2}\right)+\overline{\mathrm{H}}_{q}^{(s)}\left(\rho_{*}, \Phi_{1}\right),
$$

in view of $\overline{\mathrm{H}}_{q}^{(s)}\left(\rho_{*}, \Phi_{1}\right)=\overline{\mathrm{H}}_{q}^{(s)}\left(\sigma\left(\Phi_{2}\right), \Phi_{1} \otimes \mathrm{id}\right)$. The definition (2.12) merely gives $\overline{\mathrm{H}}_{q}^{(s)}\left(\rho_{*}, \Phi_{1}\right)=\mathrm{H}_{q}^{(s)}\left(\sigma\left(\Phi_{1}\right)\right)$, since any purification of $\rho_{*}$ is a maximally entangled state. Combining this with (5.15) completes the proof.

As in Theorem V.2 the inequalities with permutations of the three entropies also hold. Thus, the output entropy of a maximally entangled input state satisfies the two-sided estimate (5.13) for many values of the parameters $q$ and $s$. Estimation of such a kind seems to be important in the context of studies of subadditivity conjecture for a tensor product of two quantum channels [8]. The proved bounds (5.13) are expressed in terms of the two map $(q, s)$-entropies, which characterize the decoherent behaviour of involved quantum channels. For the von Neumann entropy, the inequality (5.13) was derived in Ref. [8]. Some remarks concerning the quantum Tsallis $q$-entropy are contained therein. The writers of Ref. [8] also provide another characterization of a product channel in terms of the minimum output entropies. We do not consider such entropies here.

\section{CONCLUSIONS}

We have discussed some important properties of quantum channels in terms of the unified $(q, s)$-entropies, which form a family of two-parametric extensions of the standard Shannon and von Neumann entropies. In many respects, these entropies are similar to the standard ones. For given input state, different effects of each unraveling of a channel result in some probability distribution at the output. Except for the Rényi's $q$-entropies of order $q>1$, the unified $(q, s)$-entropies of this distribution are all minimized by the same unraveling of a quantum channel. We have also specified some class of extremal unraveligs such that their $(q, s)$-entropies are bounded from below by the quantum $(q, s)$-entropy of the input state. Several upper bounds of Fannes type have been derived for the introduced map $(q, s)$-entropies. The Frobenius norm distance between two rescaled dynamical matrices is easy to express than the trace norm one. So we have given new continuity estimates of the unified $(q, s)$-entropies in terms of the Frobenius norm distance between density operators. If no of two quantum channels represents a unitary evolution then the map $(q, s)$-entropy of their tensor product is strictly subadditive in the range $\{0<q<1, s<0\} \bigcup\{1<q, 0<s\}$ and strictly superadditive in the range $\{0<q<1,0<s\} \bigcup\{1<q, s<0\}$. Extending Lindblad's inequality, we have obtained a two-sided estimate on the output $(q, s)$-entropy for the tensor product of two channels acting on maximally entangled input state. Overall, the map entropies of considered kind enjoy useful properties and may be applied in the context of quantum information processing.

\section{Appendix A: Some relations between Schatten norms}

In this appendix, we examine relations between different Schatten norms of the same operator. Few results of such a kind were presented in section IV of Ref. [33]. The statement of Lemma 3 therein allows to give upper bound on 
a unitarily invariant norm $\||X|\|$ of traceless Hermitian $X$ in terms of the trace norm $\|X\|_{1}$. In particular, we have $\sqrt{2}\|\mathrm{X}\|_{2} \leq\|\mathrm{X}\|_{1}$ for all traceless Hermitian $\mathrm{X} \in \mathcal{L}(\mathcal{H})$ [33]. In the present work, however, we are rather needed in upper bound on the trace norm in terms of the Frobenius one. In general, the following statement takes place.

Lemma A.1 Let $\mathrm{X}$ be an operator with d-dimensional support. For $p, q \geq 1$, there holds

$$
\|\mathbf{X}\|_{p} \leq d^{(q-1) /(p q)}\|\mathbf{X}\|_{p q}
$$

with the equality if and only if the $\mathrm{X}$ acts on its support as a multiple of unitary operator.

Proof. We can restrict our consideration to the support of $\mathrm{X}$, in which its singular values are non-zero. In line with the Hölder inequality, we have [13]

$$
|\langle\mathrm{u}, \mathrm{v}\rangle| \leq\|\mathrm{u}\|_{q}\|\mathrm{v}\|_{r}
$$

where the conjugate indices $q$ and $r$ obey $1 / q+1 / r=1$ and the vector norms are

$$
\|\mathrm{u}\|_{q}=\left(\sum_{j=1}^{d}\left|u_{j}\right|^{q}\right)^{1 / q}, \quad\|\mathrm{v}\|_{r}=\left(\sum_{j=1}^{d}\left|v_{j}\right|^{r}\right)^{1 / r}
$$

Putting $u_{j}=\varsigma_{j}(\mathrm{X})^{p}$ and $v_{j}=1$ for all $j$, we then get

$$
\sum_{j=1}^{d} \varsigma_{j}(\mathrm{X})^{p} \leq\left(\sum_{j=1}^{d} \varsigma_{j}(\mathrm{X})^{p q}\right)^{1 / q} d^{1-1 / q}
$$

Raising both the sides to the power $1 / p$, we finally obtain (A1). The equality in A2 takes place if and only if the $d$-tuples $\mathrm{u}$ and $\mathrm{v}$ are linearly related (see, e.g., theorem $14 \mathrm{in} \mathrm{[13]).} \mathrm{Hence} \mathrm{the} \mathrm{equality} \mathrm{in} \mathrm{(A4)} \mathrm{is} \mathrm{equivalent} \mathrm{to} \mathrm{that}$ $\varsigma_{j}=\varsigma_{k}$ for all $j \neq k$. In the basis of its eigenstates, the $|\mathbf{X}|$ is then rewritten as $|\mathbf{X}|=\varsigma_{1} \mathbb{1}$, whence the operator $\varsigma_{1}^{-1} \mathbf{X}$ is unitary.

The relation (A1) gives an upper bound on the Schatten $p$-norm in terms of other Schatten norms with larger values of the parameter. Recall that the Schatten $p$-norm is non-increasing in $p$ [1]]. In particular, for $p=1$ we have an estimate on the trace norm expressed as

$$
\|\mathbf{X}\|_{1} \leq d^{(q-1) / q}\|\mathbf{X}\|_{q}
$$

Note that the inequality (A1) remains valid for $q \rightarrow \infty$ and finite $p$, namely

$$
\|\mathrm{X}\|_{p} \leq d^{1 / p}\|\mathrm{X}\|_{\infty}
$$

This is an upper bound on the Schatten $p$-norm in terms of the spectral one. Conditions for the equality in (A6) are the same as for the equality in (A1).

[1] M. A. Nielsen and I. L. Chuang, Quantum Computation and Quantum Information. (Cambridge University Press, Cambridge, 2000)

[2] I. Bengtsson and K. Życzkowski, Geometry of Quantum States: An Introduction to Quantum Entanglement. (Cambridge University Press, Cambridge, 2006)

[3] X. Hu and Z. Ye, J. Math. Phys. 47, 023502 (2006)

[4] A. E. Rastegin, J. Stat. Phys. 143, 1120 (2011)

[5] J. S. Kim and B. Sanders, J. Phys. A: Math. Theor. 44, 295303 (2011)

[6] B. Schumacher, Phys. Rev. A 54, 2614 (1996)

[7] K. Życzkowski and I. Bengtsson, Open Sys. Inf. Dyn. 11, 3 (2004)

[8] W. Roga, K. Życzkowski, and M. Fannes, Int. J. Quantum Inf. 9, 1031 (2011)

[9] P. W. Shor, Commun. Math. Phys. 246, 453 (2004)

[10] F. G. S. L. Brandão and M. Horodecki, Open Sys. Inf. Dyn. 17, 31 (2010)

[11] J. Watrous, CS 798: Theory of Quantum Information. (University of Waterloo: http://www.cs.uwaterloo.ca/ watrous/quant-info/lecture-notes/, 2008)

[12] R. Bhatia. Matrix Analysis. (Springer, New York, 1997)

[13] G. H. Hardy, J. E. Littlewood, and G. Polya. Inequalities. (London: Cambridge University Press, 1934) 
[14] A. E. Rastegin, Quantum Inf. Process. 9, 61 (2010)

[15] A. E. Rastegin, Quantum Inf. Process. 10, 123 (2011)

[16] C. F. Dunkl et al., J. Phys. A: Math. Theor. 44, 335301 (2011)

[17] L.-M. Duan and G.-C. Guo, Phys. Lett. A 243, 261 (1998)

[18] A. Chefles and S. M. Barnett, J. Phys. A: Math. Gen. 31, 10097 (1998)

[19] A. Jamiołkowski, Rep. Math. Phys. 3, 275 (1972)

[20] M.-D. Choi, Linear Algebra Appl. 10, 285 (1975)

[21] L. V. Il'ichev, JETP 96, 982 (2003)

[22] H. J. Carmichael, An Open Systems Approach to Quantum Optics. (Lecture Notes in Physics, vol. m18) (Springer, Berlin, 1993)

[23] H. M. Wisemann and G. J. Milburn, Quantum Measurement and Control. (Cambridge University Press, Cambridge, 2010)

[24] A. E. Rastegin, J. Phys. A: Math. Theor. 44, 095303 (2011)

[25] L. P. Hughston, R. Jozsa, and W. K. Wootters, Phys. Lett. A 183, 14 (1993)

[26] J. K. Breslin and G. J. Milburn, J. Mod. Optics 44, 2469 (1997)

[27] A. Peres. Quantum Theory: Concepts and Methods. (Kluwer, Dordrecht, 1993)

[28] M. Fannes, Commun. Math. Phys. 31, 291 (1973)

[29] S. Furuichi, K. Yanagi, and K. Kuriyama, J. Inequal. Pure Appl. Math. 8(1), 5 (2007)

[30] Z. Zhang, Lett. Math. Phys. 80, 171 (2007)

[31] A. E. Rastegin, Lett. Math. Phys. 94, 229 (2010)

[32] R. Alicki and M. Fannes, J. Phys. A: Math. Gen. 37, L55 (2004)

[33] K. M. R. Audenaert and J. Eisert, J. Math. Phys. 26, 102104 (2005)

[34] A. E. Rastegin, J. Math. Phys. 52, 062203 (2011)

[35] D. Petz. Quantum Information Theory and Quantum Statistics. (Springer, Berlin, 2008)

[36] A. E. Rastegin, E-print arXiv:1102.5154 [math-ph] (2011)

[37] S. Furuichi, J. Math. Phys. 47, 023302 (2006)

[38] T. Lindvall. Lectures on the coupling method. (John Wiley \& Sons, New York, 1992)

[39] C. Tsallis, In Nonextensive Statistical Mechanics and Its Applications, eds. S. Abe and Y. Okamoto (Lecture Notes in Physics, vol. 560, Springer, Berlin, 2001), 3-98

[40] G. A. Raggio, J. Math. Phys. 36, 4785 (1995)

[41] K. Audenaert, J. Math. Phys. 48, 083507 (2007)

[42] G. Lindblad, In Quantum Aspects of Optical Communications, eds. C. Bendjaballah, O. Hirota, and S. Reynaud (Lecture Notes in Physics, vol. 378, Springer, Berlin, 1991), 71-80 